\title{
Local Tissue Growth Patterns Underlying Normal Fetal Human Brain Gyrification Quantified In Utero
}

\author{
Vidya Rajagopalan, ${ }^{1,2}$ Julia Scott, ${ }^{1,2}$ Piotr A. Habas, ${ }^{1,2}$ Kio Kim, ${ }^{1,2}$ James Corbett-Detig, ${ }^{1,2}$ Francois Rousseau, ${ }^{3}$ \\ A. James Barkovich, ${ }^{2}$ Orit A. Glenn, ${ }^{2}$ and Colin Studholme ${ }^{1,2}$ \\ ${ }^{1}$ Biomedical Image Computing Group, Department of Radiology and Biomedical Imaging, and 2Department of Radiology and Biomedical Imaging, \\ University of California, San Francisco, San Francisco, California 94143-0628, and ${ }^{3}$ Image Sciences, Computer Sciences and Remote Sensing Laboratory, \\ Unité Mixte de Recherche 7005, Centre National de la Recherche Scientifique-University of Strasbourg, 67412 Illkirch, France
}

Existing knowledge of growth patterns in the living fetal human brain is based upon in utero imaging studies by magnetic resonance imaging (MRI) and ultrasound, which describe overall growth and provide mainly qualitative findings. However, formation of the complex folded cortical structure of the adult brain requires, in part, differential rates of regional tissue growth. To better understand these local tissue growth patterns, we applied recent advances in fetal MRI motion correction and computational image analysis techniques to 40 normal fetal human brains covering a period of primary sulcal formation (20-28 gestational weeks). Growth patterns were mapped by quantifying tissue locations that were expanding more or less quickly than the overall cerebral growth rate, which reveal increasing structural complexity. We detected increased local relative growth rates in the formation of the precentral and postcentral gyri, right superior temporal gyrus, and opercula, which differentiated between the constant growth rate in underlying cerebral mantle and the accelerating rate in the cortical plate undergoing folding. Analysis focused on the cortical plate revealed greater volume increases in parietal and occipital regions compared to the frontal lobe. Cortical plate growth patterns constrained to narrower age ranges showed that gyrification, reflected by greater growth rates, was more pronounced after 24 gestational weeks. Local hemispheric volume asymmetry was located in the posterior peri-Sylvian area associated with structural lateralization in the mature brain. These maps of fetal brain growth patterns construct a spatially specific baseline of developmental biomarkers with which to correlate abnormal development in the human.

\section{Introduction}

Maturation of the human brain in the latter half of gestation is manifested by substantial increases in volume, increasing complexity of the cortical plate, and changes in the molecular and cellular composition of the cerebral mantle tissue zones. In particular, gyrification occurs in a hierarchical fashion in which primary sulci emerge in a stereotyped fashion between 10 and 28 gestational weeks, followed by secondary and tertiary sulci that show increasing variability across individuals (Dooling et al., 1983; Garel et al., 2003; Bendersky et al., 2006).

Evidence from two-dimensional (2D) in utero fetal magnetic resonance imaging (MRI) studies shows a spatially varying cortical growth pattern. Manual segmentations of cerebral mantle tissue zones on MRI describe the different growth trajectories for the overall cerebral brain, germinal matrix, and ventricular vol-

Received Oct. 18, 2010; revised Dec. 16, 2010; accepted Dec. 20, 2010.

This research was funded by the National Institutes of Health through the National Institute of Neurological Disorders and Stroke (R01 NS 061957 and R01 NS 055064); National Center for Research Resources to University of California, San Francisco-Clinical \& Translational Science Institute (UL1 RR024131); and award to 0.A.G. (K23 NS52506-03). This research was also funded by the European Research Council under the European Community Seventh Framework Programme (FP7/2007-2013 Grant Agreement 207667). We thank the participating mothers who have made this research possible.

Correspondence should be addressed to Dr. Vidya Rajagopalan, Department of Radiology and Biomedical Imaging, University of California, San Francisco, Box 0628, Slot 6, San Francisco, CA 94143-0628. E-mail: vidya.rajagopalan@ucsf.edu.

DOI:10.1523/JNEUROSCI.5458-10.2011

Copyright $\odot 2011$ the authors $\quad 0270-6474 / 11 / 312878-10 \$ 15.00 / 0$ umes (Grossman et al., 2006; Kazan-Tannus et al., 2007; Hu et al., 2009). Additionally, manual 2D measurements of laminar thickness suggest regionally varying thickness in cortex and subplate (Widjaja et al., 2010). These studies are dependent upon selective manual measurements rather than comprehensive analysis of normal brain growth. Recently, 3D reconstruction techniques for fetal MRI (Rousseau et al., 2006; Jiang et al., 2007; Kim et al., 2010) were developed together with a corresponding spatiotemporal atlas of developing brain tissues (Habas et al., 2010a). Accurate and automated delineation of developing and transient tissue boundaries (Habas et al., 2010b) allows for global and regional volumetry. However, the development of a sulcated adult brain from a smooth fetal brain requires an intricate series of local tissue volume changes to form the complex shape and cortical folding pattern (Smart and McSherry, 1986a; Hilgetag and Barbas, 2006) that underpins the functional specialization of the cortex. Local perturbations in cortical morphology are of interest as biomarkers of abnormal neurodevelopment. Here, we aim to examine the early stages of these tissue growth patterns without specific spatial hypotheses with the use of tensor-based morphometry (TBM), which examines the pattern of local size differences across a set of anatomies (Davatzikos et al., 1996; Studholme et al., 2001; Thompson et al., 2001; Aljabar et al., 2008). In this study, we use TBM analysis combined with a linear model of age to create maps that answer the question, where is tissue being added more or less quickly than the overall growth rate of the brain? 


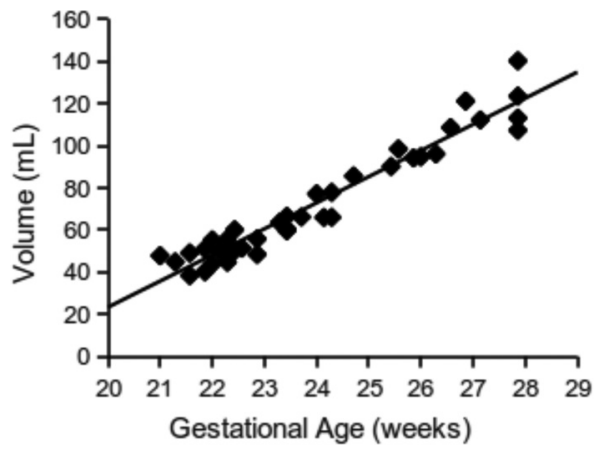

Figure 1. Estimated volume increases of the cerebrum from 20 to 28 weeks $G A$. The volume gain per week was $12.31 \mathrm{ml} /$ week, and the percentage gain relative to the group average volume (mean age 23.9 weeks $\mathrm{GA}$ ) is $17 \%$ /week.

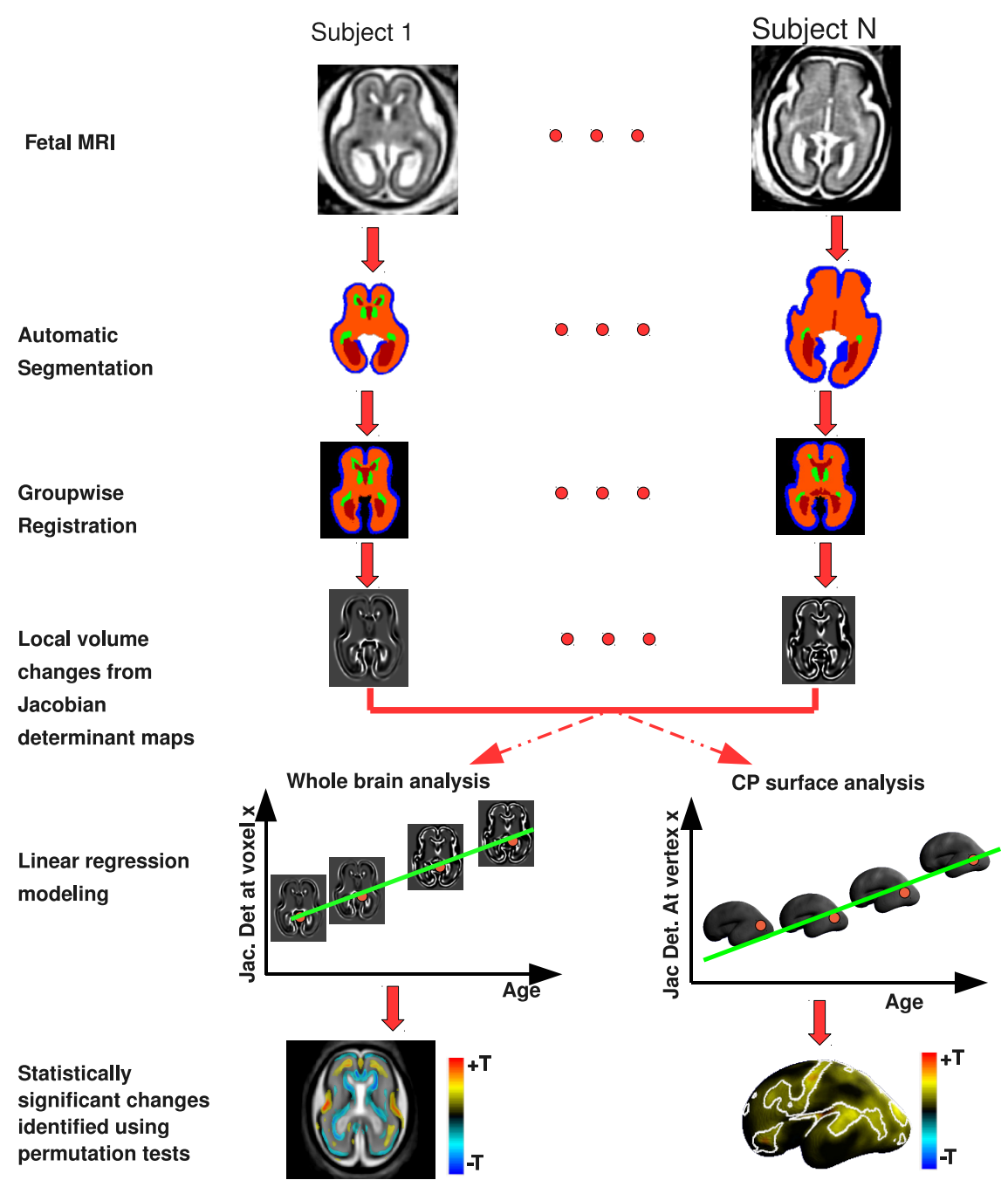

Figure 2. Graphical flowchart of the analysis procedure used to compute the growth rate maps and identify regions with significant accelerations or decelerations. The fetal MRIs of the subjects are segmented into CP; NCP, which includes subplate, intermediate zone, and deep gray nuclei; and VENT. GMAT. The segmentation maps are spatially normalized with a global affine initialization followed by groupwise registration. Jacobian determinant maps from the deformation fields are computed and are used in linear regression modeling of local volume changes with age. A t test is used to study deviations in the local growth rate relative to the overall cerebral rate. Statistical significance is computed and corrected for multiple comparison using permutation tests.

We focus on the critical period of early cortical folding, and report results of a cross-sectional study of normal fetal human anatomies imaged in utero to extract statistical models of growth patterns. As previously shown by regional volumetry (Kazan-Tannus et al.,
2007), we anticipate growth rates to differ across the zones of the cerebral mantle. We expect regions that add tissue at differential rates to be correlated with the shape changes inherent to cortical folding or reflect periods of rapid development or regression in particular structures. Knowledge of the spatiotemporal pattern of brain growth in a normal population may provide structural biomarkers for the study of abnormal brain development (Boardman et al., 2006).

\section{Materials and Methods}

Fetal subjects and image acquisition. The following experiments were performed using 40 clinical scans of 38 fetuses at gestational ages (GAs) ranging from 20 to 28 weeks GA (age distribution illustrated in Fig. 1), estimated by last menstrual period. The mothers were referred for fetal MRI due to questionable findings on prenatal ultrasound $(n=9)$ or a prior abnormal pregnancy $(n=8)$, or volunteered for scans $(n=21)$ as part of studies at University of California, San Francisco. All women had normal fetal MRI. The subject population contains 19 females and 19 males. The mean delivery age was 39.3 weeks GA (37.4-41.57 weeks GA). The imaging study has Institutional Review Board approval and complies with National Institutes of Health human subject guidelines.

Clinical MR imaging was performed on a $1.5 \mathrm{~T}$ scanner (GE Healthcare) using an eight-channel torso phased-array coil. Multiple stacks of singleshot fast spin-echo T2-weighted (T2w) slice images (in-plane pixel size of $0.5 \mathrm{~mm} \times 0.5 \mathrm{~mm}$, thickness $3 \mathrm{~mm}$, no gap) were obtained in the approximately axial, sagittal, and coronal planes with respect to the fetal brain. All slice images were acquired in an interleaved manner to reduce saturation of spins in adjacent slices. The MR sequence parameters (repetition time TR $=3000$ $9000 \mathrm{~ms}$, echo time $\mathrm{TE}=91 \mathrm{~ms}$ ) were originally designed for clinical scans.

To account and correct for spontaneous fetal movement during scanning, all image slices in the slice stacks of a subject were registered using the slice intersection motion correction (SIMC) technique (Kim et al., 2010) and reconstructed into $3 \mathrm{D}$ volumes with isotropic voxel size of $0.5 \mathrm{~mm}$. The SIMC method has been previously validated using 100 synthesized datasets and 45 clinically acquired datasets. Quantifying a level of corrected motion is difficult because we are considering the influence of the whole fetal head trajectory occurring during imaging. We have recovered trajectories where individual slices were displaced by as much as $15 \mathrm{~mm}$ or $30^{\circ}$ of rotation in one or more axes with respect to their neighbors. Although fetal motion may be expected to be highly correlated with fetal age, in a separate study of data acquired at our site, we found that maximum slice displacement did not clearly correlate with age over the age range of this study (Kim et al., 2010). The 3D volume was reconstructed by gradient-weighted Gaussian averaging, where voxels were selectively weighted by the acquisition quality and compounded into a $3 \mathrm{D}$ volume that is consistent with each of the 2D slices.

Automatic tissue segmentation. The reconstructed volumes were automatically segmented into regions of the cortical plate (CP); subplate, intermediate zone, and deep gray nuclei [not cortical plate (NCP)]; germinal matrix (GMAT); and ventricles (VENT) 
(as illustrated in Fig. 2) using the following approach. A spatiotemporal atlas of MR intensity, tissue probability, and shape of the fetal brain was created from 30 manual segmentations of subjects with gestational ages ranging from 20 to 28 weeks GA (Habas et al., 2010a). From this atlas, a synthetic age-specific MR intensity template and an age-specific tissue probability map were generated for each of the 40 scans. The subject MRI was aligned to the age-matched MR template using a sequence of global linear registrations driven by maximization of normalized mutual information (Studholme et al., 1999) followed by multiple elastic deformations driven by maximization of mutual information (Viola and Wells, 1997) within a fixed mask. Based on the inverse of the estimated spatial transformation, the agematched tissue probability map was aligned with the subject MRI and used as a source of spatially varying priors for automatic atlasbased expectation-maximization (EM) brain tissue segmentation (Habas et al., 2010b). This approach to atlas-based segmentation of developing tissues in the human brain had been previously validated for young fetal subjects with normal brain development (Habas et al., 2010a, Habas et al., 2010b). The average values of Dice similarity coefficient (DSC) between automatic and manual segmentations calculated for 30 fetal anatomies were $0.88 \pm 0.03$ for $\mathrm{CP}, 0.95 \pm 0.01$ for NCP, $0.80 \pm 0.04$ for GMAT, and $0.90 \pm 0.03$ for VENT.

Groupwise registration. To compare anatomies at different stages of development in detail, we need to estimate a mapping that brings each subject's anatomy into correspondence with a common anatomical coordinate system. Unlike adult morphometric studies (Thompson et al., 2000; Studholme et al., 2004; Cardenas et al., 2007; Pieperhoff et al., 2008), because of the significant range of anatomies being studied here, we have used a template-free approach (Studholme and Cardenas, 2004) to avoid the introduction of shape bias into the analysis. To do this, the tissue label maps for each of the 40 scans were coaligned using an unbiased groupwise registration algorithm (Fig. 2). The algorithm simultaneously estimated an average brain shape and a deformable mapping to each of the anatomies being studied.

This average shape was estimated in such a way to ensure that the average distance from each point in that space, when mapped to the individuals in the group, is forced to be zero, forming a so-called minimum deformation anatomy. To avoid the additional possibility of bias caused by significant MR tissue contrast change with age in the developing fetal brain, we took the approach of aligning tissue label maps derived from automated segmentation, rather than aligning the raw MR image values directly, as is commonly used in adult TBM studies (Chung et al., 2001), where tissue contrast can be assumed to be constant. Finally, we also know that some transient tissue classes are not consistently present over the age range of our study, specifically regions of GMAT and subplate are present at the beginning of this period ( 20.5 weeks GA) but are not visible in MRI in many brain regions later in the developmental period (27 weeks GA). As a result, estimating a meaningful diffeomorphic mapping for a TBM analysis would not be possible with these inconsistent boundaries, since these would induce artifactual folding of the mapping between anatomies as the alignment attempts to account for the missing tissue classes in older anatomies. In this study, we therefore took the approach of combining the tissue classes, so as to exclude inconsistent transient tissue boundaries from the registration process. This was achieved by combining the transient tissue classes, corresponding to the germinal matrix, the intermediate zone, subplate, and subcortical gray matter, into NCP tissue label.

For the alignment of the different anatomies, we first seeded the groupwise registration using a linear registration of the NCP regions of each subject to a single average NCP tissue map as in (Habas et al., 2010a). We then estimated a set of unbiased dense field deformations that minimize the mean square difference between each subject tissue label map (NCP, CP, CSF) and the current average (NCP, CP, CSF) tissue label map of the group. This was iteratively refined using a gradient descent of the squared difference in tissue labels between the current average and each individual's tissue map, summed over each tissue class, then minimized with respect to the displacement vector at each voxel. A Gaussian smoothing operator (Thirion, 1998; Modersitzki, 2004) was used to regularize the deformation fields during alignment to ensure a spatially differentiable mapping. A composition of a sequence of two of these diffeomorphic mappings, each characterized by a vector field of displacements, from the average anatomy to each subject was estimated. This first captures larger-scale shape differences (using a $2 \mathrm{~mm}$ Gaussian regularization kernel) and then more local shape differences (using a 1 $\mathrm{mm}$ Gaussian regularization kernel). These regularization factors were chosen experimentally to enforce numerically diffeomorphic mappings during the registration. To validate this process, we used a subset of subjects with a representative age distribution for which manual tissue segmentations were also available. First, this group was aligned using the combination of automated tissue segmentation and groupwise alignment used in this paper. Then the transformations were used to map the manual segmentations into the resulting average space, allowing an independent measure of tissue alignment. The resulting overlap of the manually segmented regions in the common space was evaluated with DSC coefficients of $0.84 \pm 0.02$ for the $\mathrm{CP}$ and $0.94 \pm 0.01$ for the NCP. This was comparable to the underlying differences between manual and automated tissue segmentation, indicating that the groupwise registration had resolved the majority of true differences in anatomy. In addition, we confirmed that all deformations remained diffeomorphic for these regularization parameters.

Deformation tensor morphometry and statistical analysis. The resulting Jacobian matrix of the sequence of deformations at each point in the reference space to each subject was then calculated (Christensen et al., 1996) and used to provide a map of the local size differences between the average anatomy and each individual anatomy of the group. The determinant of this matrix provided a scalar summary of the volume changes in three spatial axes (Fig. 2). An illustration of the Jacobian determinant map for a representative subject is shown in Figure 3. 
a

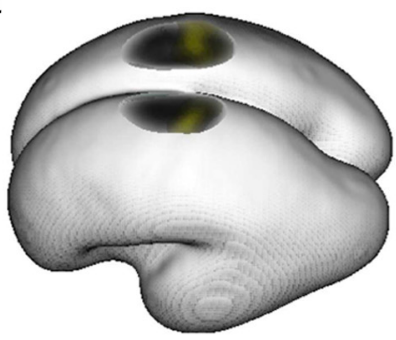

d

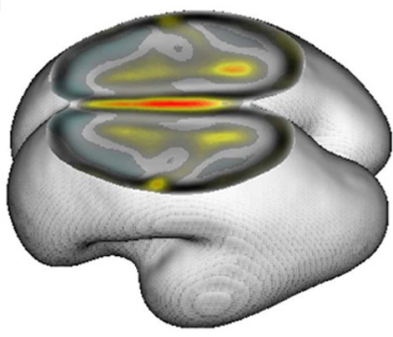

g

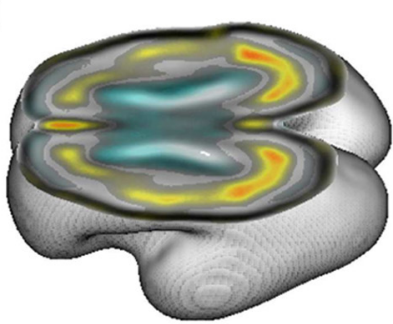

j

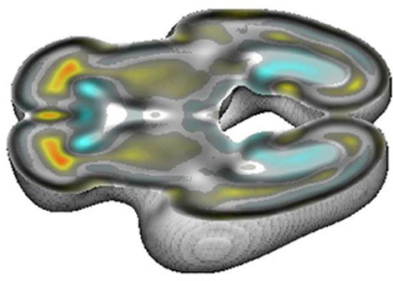

m

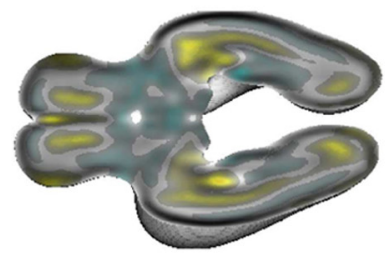

b

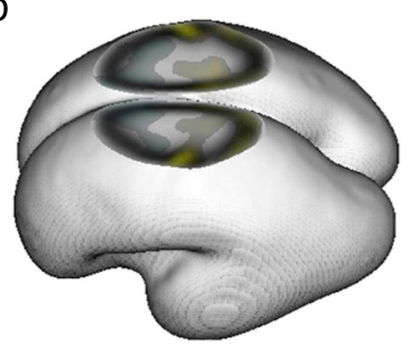

e

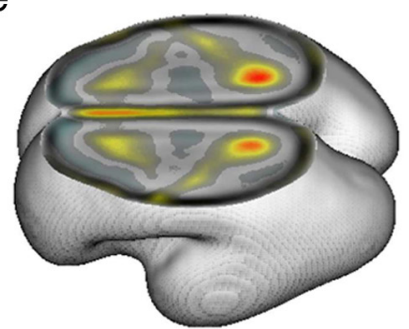

h

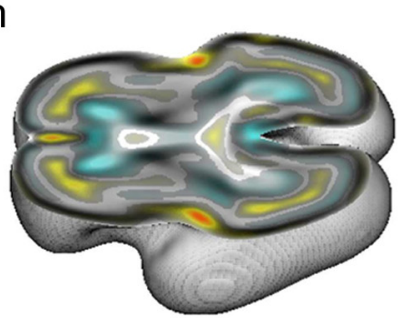

k

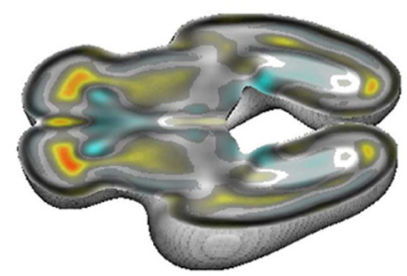

$\mathrm{n}$

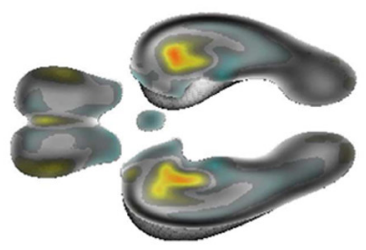

C

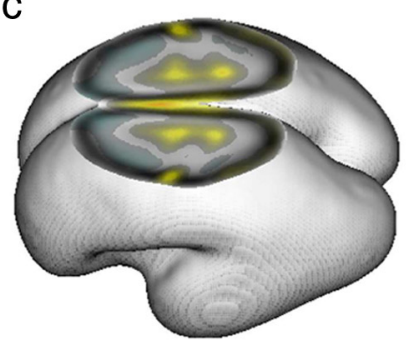

f

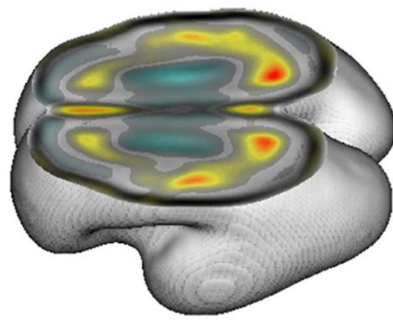

i

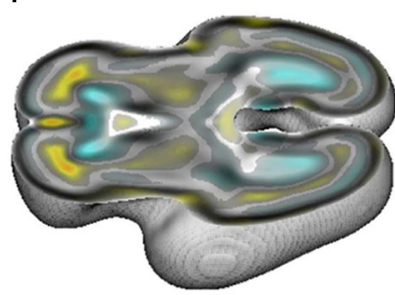

I

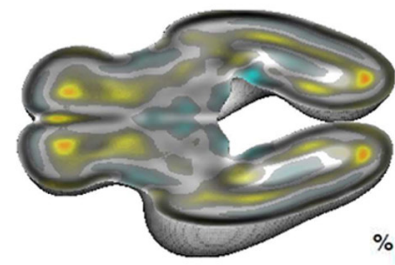

$\%$ per week

0

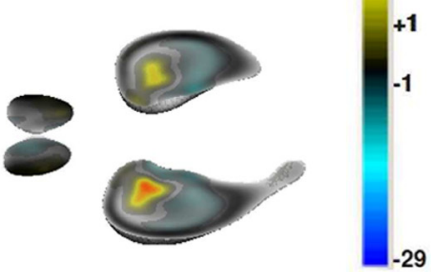

Figure 4. Local tissue growth rate patterns relative to cerebral growth rate, overlaid on the average brain. The color scale represents the growth rate per week for each voxel. Figure panels display axial sections from superior to inferior at $5 \mathrm{~mm}$ intervals. The growth rate varied across the zones of the cerebral mantle.

We analyzed the set of maps of the subjects using voxelwise general linear modeling with age, to test the hypothesis that the relationship between the local tissue volume changes with age (i.e., growth rate) is significantly different (greater or lesser) than the overall cerebral growth rate. Here we determined the overall cerebral growth rate by the size changes captured by the initial linear registration together with the average Jacobian of the cerebral tissues for each subject. We performed two forms of shape analysis derived from the deformation tensor maps (Fig. 2): first, a voxelwise analysis covering all voxels in brain tissues to create 3D statistical map of growth, and then one specifically focused on the cortical plate by forming 2D maps over the brain surface to provide increased sensitivity by constraining the statistical analysis to the cortex. To perform a cortical surface analysis, the outer surface of the cortical plate in the group average tissue segmentation map was formed into a triangulated mesh using a topology preserving marching cubes algorithm (Lopes and Brodlie, 2003). The Jacobian determinant values representing changes in local thickness and area of the cortical plate within 4 $\mathrm{mm}$ of each surface vertex were averaged and placed onto the surface mesh for surface modeling. For each subject, this then provided a map that summarizes underlying changes in both surface area and thickness of the outer CP.

For both voxel and surface analyses, a linear model against age was constructed to test our hypotheses (Fig. 2). This formed a linear regression (using all scans) with age as the independent variable and the Jaco- 
bian determinant (after correction for total cerebral size) as the dependent variable. The resulting regression coefficients $(B)$ provided an estimate of the local rate of growth relative to the whole cerebrum. $T$ statistics were calculated for each voxel or surface vertex together with the corresponding multiple-comparisons-corrected significance values (corrected using nonparametric permutation testing) (Nichols and Holmes, 2002).

Changes in relative growth rate and pattern over time. We initially performed a constant linear growth model for our voxelwise analysis, though we were interested in the changes in the rate of relative tissue growth. However, statistically and computationally we were limited by the sample size for a full voxelwise nonlinear analysis. Therefore we chose to look for changes in rate by dividing the group by age and estimate linear models in each. The subgroups were more mature (17 scans with $\mathrm{GA} \geq 24$ weeks such that age distribution is $26.03 \pm 1.04$ weeks GA) and less mature ( 23 scans with GA $<24$ weeks such that age distribution is $22.31 \pm 0.82$ weeks GA). Groupwise registration was repeated for each group separately and growth patterns (over both whole brain and the CP region) were analyzed as described previously. By analyzing the two groups individually, we examine changes in the growth pattern over time. Qualitative MRI studies typically detect sulci 2-3 weeks later than found by histopathology (Dooling et al., 1983; Garel et al., 2003). For example, the central, collateral, and superior temporal sulci are visible by 23 weeks GA on histology, but after 26 weeks GA on MRI (Garel et al., 2003). Here, we examine whether TBM allows us to identify emergence of early primary sulci before they are known to become visually detectable on the MRI.

Quantifying regional growth rates. We next performed a secondary analysis of regions with significant deviations from the cerebral growth rate to explore whether the rate of growth in these regions was better modeled nonlinearly. However, explicit nonlinear modeling of changes in the relative growth rate at each voxel is limited by the number of subjects in our study. Therefore, we used the initial voxelwise statistical maps to identify distinct subregions among the significantly faster growing voxels. We then located highly significant voxels (with $T \geq 10$ ) and used these as region centers. For every voxel with $T>5.86(p<0.05$, corrected), we assigned it to the nearest connected region center to create a partitioning of the growth pattern. The regions of interest (ROIs) corresponded to anatomical regions that contribute to the most significant local variations in growth rate. Using all subjects, we computed the average Jacobian determinant value within each ROI, which gives a summary measure of how that region of tissue changes in size relative to the cerebrum. Growth in each ROI was then represented as a percentage change per week relative to the cerebral growth rate. For larger regions with volume $\geq 0.2 \mathrm{~cm}^{3}$, we also computed the local growth rate by using a best-fit linear or nonlinear model with the average Jacobian determinant as the dependent variable and age as the independent variable. These models allowed us to quantify the rates at which tissue is being added at specific anatomical locations in the brain within groups of voxels that are growing together, rather than enforce a priori spatial hypothesis of manually delineated regions.

Local volume asymmetry. To examine the emergence of asymmetries, we performed an additional TBM analysis that used a symmetric groupwise nonrigid registration of the tissue maps that also accounted for differences in anatomy on each side of the brain. This was performed by reflecting each of the tissue maps along the sagittal midline (longitudinal fissure) and then registering the original and reflected brains collectively to form a single symmetric average brain shape from the group. We then tested for age-consistent size differences between the left and right hemispheres, while accounting for linear growth rate in the group. This analysis was applied to the whole group and then separately to the less and more mature groups to look for changes in asymmetry. Permutation testing was used within a brain mask of the right hemisphere to correct the asymmetry maps of significance for multiple comparisons. Positive significant hemispheric differences in local volume indicated greater volume in the right hemisphere compared to the left and vice versa for negative values.

\section{Results}

In overview, we detected volumetric patterns of significantly ( $p<0.05$, corrected) slower or faster linear growth than the overall cerebral growth rate as determined from voxelwise and
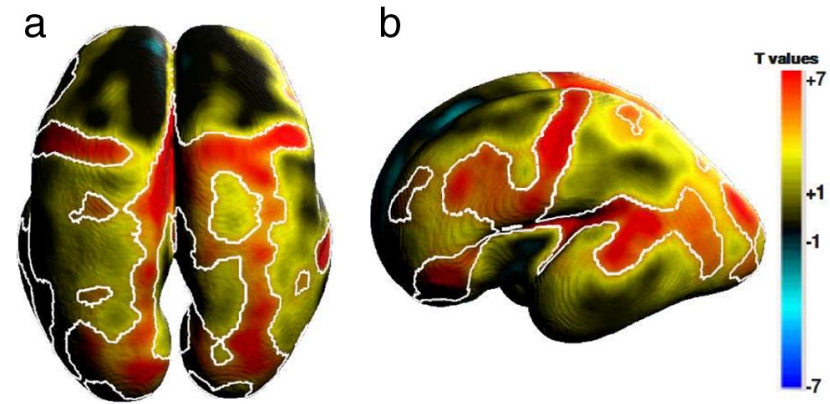

Figure 5. Cortical growth rate patterns. $\boldsymbol{a}, \boldsymbol{b}$, Dorsal ( $\boldsymbol{a}$ ) and lateral $(\boldsymbol{b})$ views of $T$ statistic map of the model of $(P$ volume increases. White contours indicate regions that are statistically significantly different from the cerebral growth rate after multiple-comparisons correction $(p<0.05)$. Mostly positive $T$ values indicate faster than cerebral growth rate across the majority of the $C P$.

surface-based analyses. Reported local rates are percentage volume change per week relative to the average cerebral growth rate of $17 \%$ per week (Fig. 1). For example at a particular voxel or vertex, $-6 \%$ means that this location grew $6 \%$ per week less than the average weekly rate of $17 \%$, or an absolute rate of $\sim 11 \%$ per week. Voxelwise results are presented in order of increasing depth of the brain: cortical plate, remaining fetal brain tissue zones and subcortical nuclei (NCP), and ventricles. Significant changes in volume are predominantly bilateral unless noted otherwise. $T$ statistic maps displayed in each orthogonal plane and minimum and maximum intensity projections are included in supplemental material (available at www.jneurosci.org).

In this TBM study, folding of the cortical plate would appear as a greater expansion rate at sulcal fundi because the tissue is being warped away from the corresponding voxel on the average brain. Therefore our interpretation of faster growth in the cortical plate at anticipated sulcal sites reflects the additional infolding of the sulci relative to the outer skull boundary. Without additional image contrasts that provide cortical landmarks [e.g., diffusion tensor imaging (DTI)] to define corresponding regions of cortex at different developmental ages, these maps cannot directly examine differences in growth rate between gyral crests and sulcal valleys as histological studies may be able to (Hilgetag and Barbas, 2006; Bystron et al., 2008).

For the voxelwise analysis, growth rate maps showing regions of significantly increased or decreased growth (compared to the overall growth) are overlaid on the spatially normalized average MRI (Fig. 4) and displayed using the RView software (http://rview.colin-studholme.net). $T$ value surface maps computed from the analysis of the CP surfaces are used to indicate differential growth patterns on the CP with contours delineating regions of statistically significant local variations in growth rates (Fig. 5). Mean relative growth rates are reported in Table 1 and Figure 6 for regions that exhibit significantly greater growth rate than average cerebral growth rate. Differences in relative growth rates between less and more mature groups are studied by comparing their individual regression analyses (Figs. $7,8)$. Last, we tested for local volume differences across hemispheres over the full age range and the age subgroups (Figs. 9, 10).

\section{Local relative growth patterns}

Cortical plate

The deformation tensor model localized to the cortical plate is sensitive to focal changes in both cortical thickness and area. Local growth in the cortical plate was either similar to or significantly greater than the overall cerebral rate (Fig. 1). Quantitatively, from the regional analysis, the local relative growth rates 
Table 1. Average regional growth rates (relative to cerebral growth rate) for the most significant regions

\begin{tabular}{llcl}
\hline Regions & Hemisphere & $\begin{array}{l}\text { Rate } \\
(\% / \text { week })\end{array}$ & $\begin{array}{l}\text { Volume } \\
\left(\mathrm{cm}^{3}\right)\end{array}$ \\
\hline Anterior frontal NCP & Left & 9.63 & 0.27 \\
Anterior frontal NCP & Right & 11.00 & 0.16 \\
Anterior temporal NCP & Left & 12.90 & 0.14 \\
Anterior temporal NCP & Right & 12.27 & 0.07 \\
Anterior thalamus & Right & 7.37 & 0.02 \\
Central sulcus & Left & 17.94 & 0.02 \\
Central sulcus & Right & 18.89 & 0.03 \\
Cingulate sulcus & Both & 11.99 & 0.79 \\
Frontoparietal NCP & Left & 10.45 & 0.55 \\
Frontoparietal NCP & Right & 9.90 & 0.95 \\
Hippocampus tail & Right & 14.09 & 0.05 \\
Insula and operculum & Left & 11.84 & 0.67 \\
Insula and operculum & Right & 11.10 & 0.84 \\
Orbital sulcus & Left & 10.63 & 0.03 \\
Superior temporal sulcus & Right & 9.73 & 0.06 \\
Temporal operculum & Left & 10.41 & 0.12 \\
Ventral caudate & Left & 6.57 & 0.13 \\
Ventromedial occipital NCP & Right & 15.56 & 0.02 \\
\hline
\end{tabular}

a

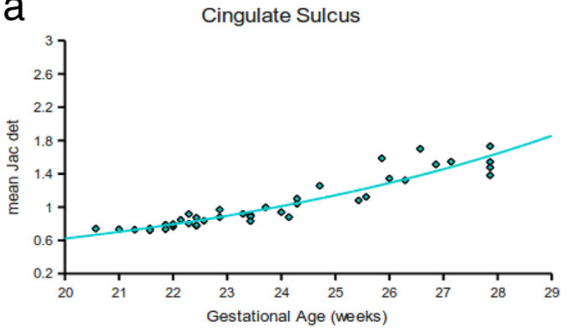

b

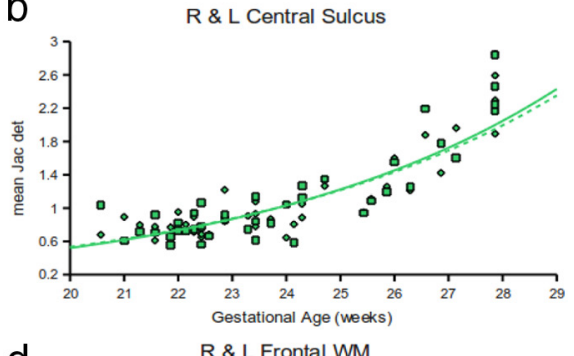

C

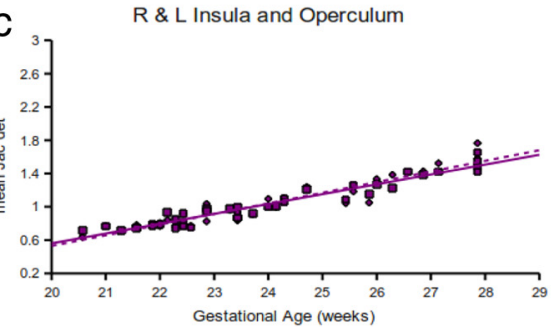

d

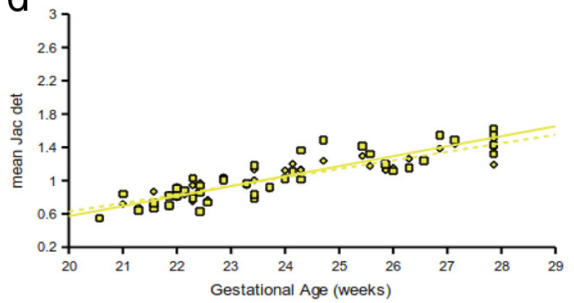

e

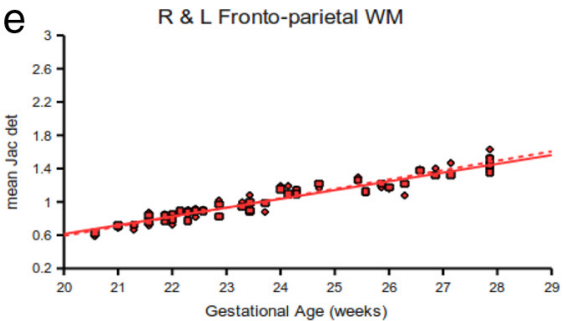

f

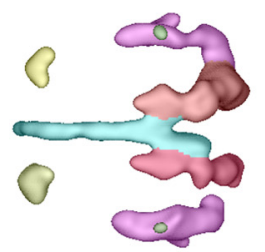

Figure 6. Regional analysis of growth rate changes for bilateral ROls with volume $\geq 0.2 \mathrm{~cm}^{3} . \boldsymbol{a}-\boldsymbol{e}$, Mean Jacobian determinant calculated for each significant ROl, for all subjects mapped against age of the subject. Each panel shows the best-fit model against age for each ROI (right, solid; left, dashed): combined right and left cingulate CP (blue) (a), right (square) and left (diamond) central sulci $C P$ (green) (b), right and left insula and operculum CP and NCP (purple) (c), right and left frontal NCP (yellow) (d), and right and left midline frontoparietal NCP (red) (e).f fand $\boldsymbol{g}$ are 3D surface representations of significant ROIs. The color of each ROI corresponds to the color used for the growth rate plot lines $(\boldsymbol{a}-\boldsymbol{e})$.

varied in that the greatest deviations were up to twice the global rate (Table 1, Fig. 6). Similar patterns in the cortical plate were found in the voxelwise and surface analyses, though larger regions of significant change were detected by the models specifically constrained to the surface (Fig. 5). The most rapid growth in the cortical plate occurred along the midline surface of the frontal g

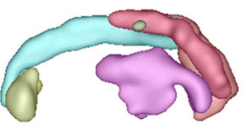

and parietal lobes (Fig. 4b). Posterior to the central sulcus, the dorsal and lateral cortical plate expanded faster than the overall cerebral rate (Figs. $4 b-h, 5 a$ ). Specifically, increased growth was apparent over most of the insula and frontal, parietal, and temporal opercula (Fig. 5b).

Other areas of significantly greater growth were found bilaterally at emerging sulci: central (Figs. $4 a-e, 5 b, 6 b$ ), circular (Figs. $4 h-k, 5 a$ ), and cingulate (Fig. $4 c-g$ ). From the quantitative regional analysis, the central and cingulate sulci expanded at the greatest relative rate compared to other locations in the cortical (Table 1). However, the regional growth rate along these linear rate (Fig. $6 a, b$ ), which indicates an acceleration of growth in these regions. To a lesser degree, the lateral orbital (Fig. $4 l-n$ ), (Fig. 4h-j), right parieto-occipital (Fig. 4j), and right calcarine (Fig. 4i) sulci grew at increased rates.

Fetal brain tissue zones and subcortical nuclei

In large uniform regions of the fetal brain tissue zones (such as deep within the cerebral mantle), the available contrast of T2w MRI (and the derived NCP segmentation) limits our ability to localize volume changes to specific tissue zones. As a result, TBM studies of patterns of tissue expansion or contraction occurring within these regions must be interpreted with care. However, by using an optimally smooth regularization of the deformation fields, the observed volume increases or decreases in these cases correspond to regions where surrounding tissue boundaries are consistently moving away or toward the center of growth, respectively.

Relative growth rates in the NCP varied spatially. The greatest regional growth rates, $\sim 11 \%$ greater than overall brain growth, were located in the anterior frontal and temporal lobes and the opercula (Table 1). Importantly, increased relative growth was found in areas associated with gyrification: operculum (Fig. $4 f-l$ ), superior temporal gyrus (Fig. $4 m$ ), precentral and postcentral gyri (Fig. $4 b-d$ ), parietal lobules (Fig. 4e,f), and ventromedial occipital lobe (Fig. 4l,m). In contrast to subplate and intermediate zone deep to the developing operculum, regions deep to the insula expand at a slower rate (Fig. $4 h-$ l). The dorsomedial aspect of the subplate and intermediate zone in the frontal and the parietal lobes (Fig. $4 b, c, e-g$ ) and the anterior frontal cerebral mantle (Fig. $4 h-l)$ grew faster than the overall cerebral rate. Near 20 weeks GA, the relative size of these cerebral mantle areas to the average brain was similar, as shown by the growth curve intercept (calculated from regional analyses) in Figure 6. Further calculation of the regional mean rates show that these are growing linearly with age (Fig. $6 c-e$ ).

Some regions of subcortical nuclei displayed significantly greater than cerebral growth rates. The ventral part of the basal ganglia showed elevated relative growth rates (Fig. $4 j-l$ ) as well a region localized to the anterior thalamus (Fig. $4 i$ ). The hip- 
a

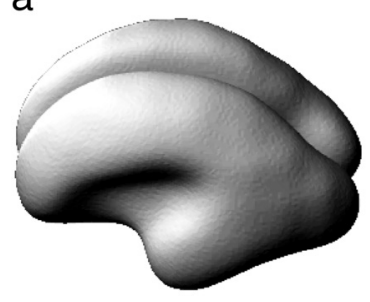

b

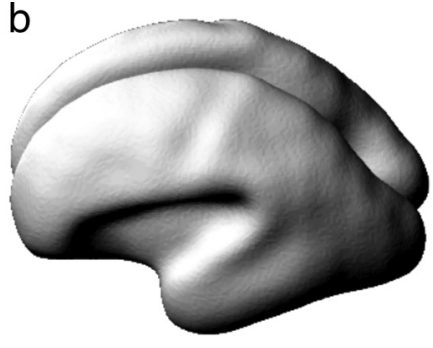

Figure 7. Surface rendering of average shape of the inner $\mathrm{CP}$ for $20-23$ weeks $\mathrm{GA}(\boldsymbol{a})$ and $24-28$ weeks $G A(\boldsymbol{b})$. The progression of folding is clearly visible, with primary sulci discernible on the average inner $\mathrm{CP}$ surface in $\boldsymbol{b}$.
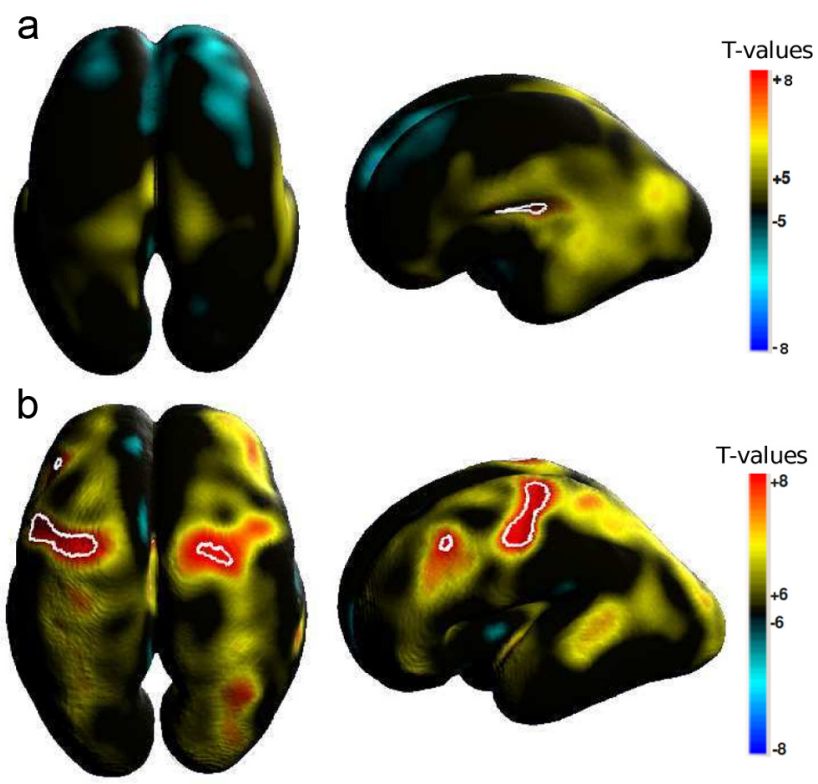

Figure 8. Maps showing the changes in the relative linear growth pattern over time between the more and less mature groups. $T$ statistic map of $(P$ growth rate relative to overall cerebral brain growth, overlaid on average the CP surface for the less $(\boldsymbol{a})$ and more $(\boldsymbol{b})$ mature age groups. White contours indicate regions that are statistically significantly different from overall cerebral brain growth after multiple-comparisons correction $(p<0.05)$. Before 24 weeks $G A$, significantly greater than average growth is seen along the left frontoparietal operculum. The superior part of the central sulcus grew at an accelerated rate compared to average growth from 24 and 28 weeks $G A$.

pocampus showed accelerated growth in the tail bilaterally and in the right anterior part (Fig. $4 k-m$ ). Reduced growth rates were localized to the basal forebrain (Fig. $4 l, m$ ).

Decreased relative growth rates were observed in the NCP, close to the NCP-VENT boundary, corresponding to the germinal matrix bilaterally in frontal (Fig. $4 f-j$ ), parietal (Fig. $4 f-h$ ), and occipital (Fig. $4 h, i)$ lobes. In the frontal lobe, only the germinal matrix surrounding the horns of the lateral ventricles had a lower growth rate. In the parietal lobe, the germinal matrix around the lateral ventricles, and not the subcortical nuclei, showed significantly lower relative growth rates. No areas of the germinal matrix exhibited greater than cerebral growth rates.

\section{Ventricles}

The boundary of the ventricles shared with the cerebral mantle showed decreased relative growth rate (Fig. $4 g-j$ ). The ventricles did not have any areas of relatively greater growth rate than the whole cerebrum.

Relative growth patterns before and after 24 weeks GA

The mean NCP surfaces of the average shape of the less and more mature groups are shown in Figure 7 and demonstrate the increased volume and development of cortical folds with age in the two groups. In the TBM analysis, as a consequence of smaller group sizes and conservative multiple-comparisons correction, fewer voxels reached significance in the analysis within the age groups, and the patterns exhibited less similarity in both hemispheres compared to the results from the full age range (Fig. 8). Despite this, the patterns in the relative growth rate maps of the more mature group were similar to that of the full age range (Fig. $8 b$ ). In the latter age group, increased relative growth rates were localized to the central, cingulate, and superior temporal sulci and dorsal parietal and occipital lobes. Relative to the average growth rate from 24 to 28 weeks GA, the opercula had a lower growth rate, which was unlike the broader age range.

The pattern of growth in the less mature group qualitatively matched that of the full age range, in that reduced relative growth rates were present in much of the dorsal frontal lobe (Fig. 8a). In contrast to the more mature group, the posterior operculum and insula grew at a slightly faster rate in the less mature group, which is also represented in the full age range.

\section{Hemispheric volume asymmetry}

In some regions of accelerated growth, the volume was not bilaterally symmetrical. Specifically, the opercula showed a distinct asymmetry in which the left posterior frontoparietal operculum was larger than the right and the right posterior temporal operculum was larger than the left (Fig. 9a). As shown by asymmetry analysis of the less and more mature age groups, the location of significant asymmetry shifted posteriorly with age (Fig. $9 b, c)$. The difference in the local volume is illustrated in Figure 10, in which the original and symmetrical average cortical plate are overlaid. The frontal and postcentral dorsomedial cerebral mantle, including cortical plate, was larger in the right hemisphere (Fig. 9a) and did not include the frontal pole. This pattern was also found when the more mature group was analyzed alone (Fig. $9 c$ ), whereas in the less mature group, right greater than left asymmetry was primarily localized to the presumptive precentral gyrus (Fig. $9 b$ ).

A small area of the left parahippocampal cerebral mantle was larger on the left compared to the right, and this asymmetry was present in the age subgroups. In the analysis of the entire age range, the ventromedial cerebral mantle of the occipital pole was larger in the left hemisphere, but this was not detected in either subgroup. In addition, an area of the caudate body was larger in the right hemisphere when the whole group was analyzed, but not in either subgroup. In a ventrolateral part of the cerebral mantle of the posterior temporal lobe, there was a localized region that was larger on the left hemisphere, though this did not correspond to a distinct anatomical location. In the less mature group only, the tail of the hippocampus was larger in the left hemisphere. There were not any regions of significant asymmetry found in the more mature group alone.

\section{Discussion}

In this work we have created, for the first time, maps of the local variation in tissue expansion in the normal human fetal brain in utero to examine the emergence of structural complexity. The age range studied (20-28 weeks GA) covers a developmental stage from a time at which few sulci have formed to a time at which most of the primary sulci have formed, but before secondary sulci appearance on MRI (Garel et al., 2003). This age range is also important clinically, since this coincides with the typical gestational age for clinical MRI scans, when critical evaluations of the 

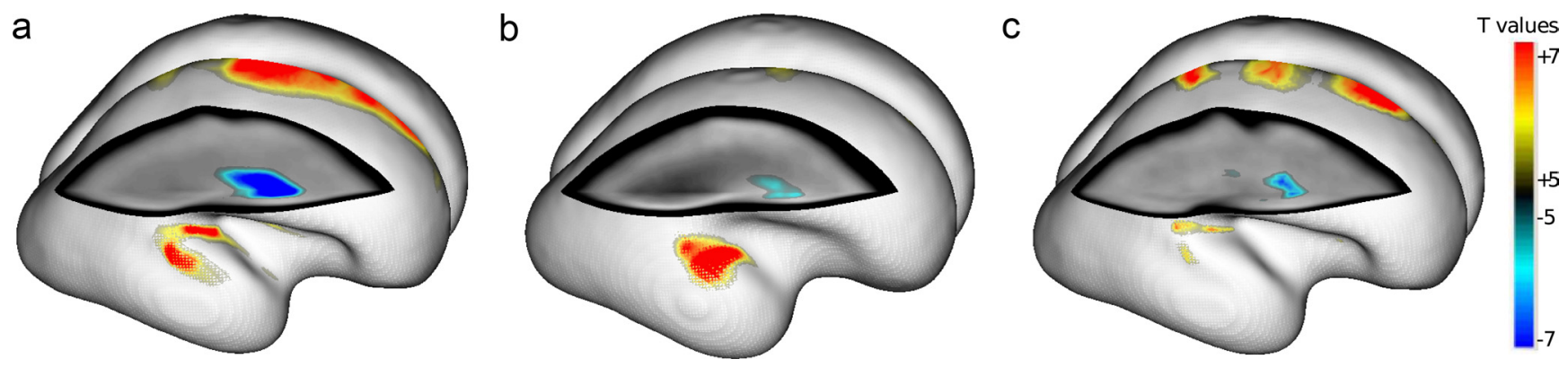

Figure 9. Tstatistic maps of significant ( $p<0.05$, corrected) local volumetric asymmetries present from 20 to 28 weeks $\mathrm{GA}$, in which warm colors indicate right hemisphere greater than left and cool colors represent left greater than right. $T$ values are projected on the surface of the brain and overlaid on cross-sectional slices of the right hemisphere. The peri-Sylvian and dorsomedial asymmetric regions are illustrated in the full age range $(\boldsymbol{a})$, less mature $(\boldsymbol{b})$, and more mature $(\boldsymbol{c})$ groups. The asymmetry around the Sylvian fissure was concentrated to the posterior extent during this opercularization process. The volume of rightward asymmetry in the frontal and parietal lobes increased with age as shown by the small pericentral region in the less mature group compared to the much larger extent in the more mature group.

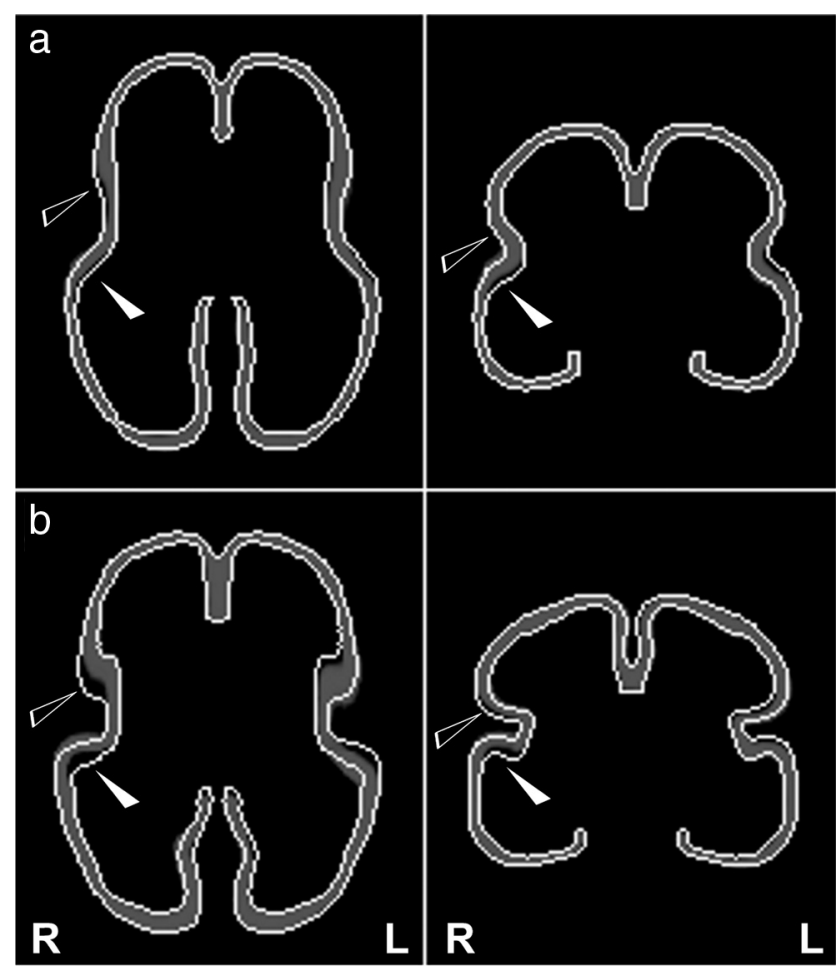

Figure 10. Cross-sectional images of the $C P$ mask and symmetrical contour of the average brains from 20 to 23 weeks $G A$ ( $\boldsymbol{a}$ ) and 24 to 28 weeks $G A(\boldsymbol{b})$ shown in the axial (left) and coronal (right) planes. The asymmetry in the peri-Sylvian region is clearly visible in the more mature average brain, which demonstrates that the asymmetry becomes more pronounced with age (solid arrow, temporal operculum; open arrow, parietal operculum).

pregnancy are often needed (Bendersky et al., 2006; Glenn and Barkovich, 2006). Normal growth patterns of this period are therefore vital for defining delay or abnormality in the formation of sulcal and gyral structures (e.g., Guibaud et al., 2008). Our findings highlighted the development of primary sulci, by the locations of significant volume changes, that are known to appear between 20 and 28 weeks GA, namely, the central, cingulate, circular, calcarine, and superior temporal sulci (Dooling et al., 1983; Garel et al., 2003). Relative growth in the fetal brain was spatially heterogeneous and associated with specific tissue zones. Generally, faster than average growth was localized to emerging sulci and the subplate and intermediate zone. On the other hand, slower growth was found in the germinal matrix and ventricles. These spatiotemporal patterns derived from the relative growth rate maps cannot be directly compared to previous qualitative and quantitative studies that do not have comprehensive local measurements. So, we will discuss how these results fit into the existing knowledge based upon global MRI morphological analyses and developmental time courses from histopathology of the human fetal brain.

\section{Comparison to previous findings}

We have detected the formation of sulci by a voxelwise model of local volume changes over time, in contrast to visual inspection that qualifies only the presence or absence of a sulcus. This means we could identify the period of time in which a change in shape was occurring. By calculating mean relative growth rate in anatomically meaningful regions, we have differentiated between the growth patterns in subplate and intermediate zone and the accelerating rate in the cortical plate undergoing folding (Fig. 6). This may reflect the developmental processes of increasing complexity and transient connectivity in the subplate and the beginning of corticocortical and thalamocortical connectivity leading to gyrogenesis (Hilgetag and Barbas, 2006; Diaz and Gleeson, 2009; Kostović and Judas, 2010). The timing of the differential growth patterns corresponds to what is known about the time course of cortical connectivity. For example, we show that the accelerating changes at the central sulcus were concentrated to after 24 weeks GA (Fig. 8), which overlaps with the arrival of thalamocortical input to somatosensory cortical plate (Kostovic and Rakic, 1990).

The relative growth rate maps show how growth of multiple tissue zones is associated with morphological changes of the cortical sheet. Global measures of volume (e.g., ROIs) or curvature (e.g., gyrification index) alone would not have revealed the spatial relationship between tissues since they do not localize where the tissue-specific changes are occurring. For example, $\mathrm{Hu}$ et al. (2009) did not find strong correlations between regional cerebral volume growth rates and regional curvature index between 21 and 37 weeks GA. However, global analysis of volume and gyrification show that acceleration of growth occurs at the same time as primary gyrification (Kochunov et al., 2010). By studying similar parameters (volume and shape) in a voxelwise manner with TBM, we have shown that tissue volume is expanding in NCP under gyral crests adjacent to sites of sulcation, which was indicated by increased relative growth rates in the cortical plate at sulcal fundi (Fig. 4c; supplemental Figs. 12, 13, available at www.jneurosci. org as supplemental material). The associated volume changes between tissue zones have not previously been demonstrated in the human fetal brain in utero, but been proposed in histological studies of other species (e.g., Smart and McSherry, 1986b). 
Like visual inspection-based MRI studies of gyrification, our TBM analysis also detected the emergence of most primary sulci after 24 weeks GA. Application of TBM to the less mature group did not identify significant changes in some sulci, such as the superior temporal sulcus, which are also not identified in qualitative MRI studies at this age (Garel et al., 2003; Bendersky et al., 2006). However, histopathological studies do locate the superior temporal sulcus in $75 \%$ of cases by 23 weeks GA (Dooling et al., 1983). The disparity between what can be visualized in T2w MRI compared to histopathology at this fetal stage suggests limitations in the resolution and contrast of in utero MRI studies. Additionally, TBM analysis is not sensitive to sulci that are developing at a rate similar to the average growth of the brain. These may include the callosal and olfactory sulci, which are identified before 20 weeks GA on histopathological sections (Dooling et al., 1983).

By comparing the local volume differences across hemispheres, we have shown that peri-Sylvian asymmetries are emerging by 20 weeks GA (Toga and Thompson, 2003). This is demonstrated by the greater volume in the right posterior temporal operculum, which would be associated with the sharper angle of the posterior Sylvian fissure on the right hemisphere (LeMay, 1976), resulting in relatively more tissue in this region on the right hemisphere. The leftward parietofrontal operculum asymmetry was not anticipated (Toi et al., 2004; Quarello et al., 2008) and may be due to morphological characteristics associated with the Sylvian fissure path that shift the relative position of the tissue (Fig. 10). Interestingly, the rightward asymmetry in the medial frontal lobe and leftward asymmetry in the medial occipital pole may be evidence of the beginning of hemispheric frontal and occipital torque, which is present in the neonatal brain (Tzarouchi et al., 2009). These findings suggest that some hemispheric asymmetries occur as the structures form.

Growth in the germinal matrix and ventricles was significantly slower than cerebral growth from 20 to 28 weeks GA. Previous volumetric studies indicate that little, if any, absolute increase in germinal matrix size occurs after 20 weeks GA (Jammes and Gilles, 1983; Kinoshita et al., 2001). The current TBM analysis adds that the reduced relative growth rate is consistent across much of the germinal matrix. Planimetry analysis of postmortem fetal brain tissue shows that both the ganglionic and extraganglionic eminences reach maximum volume at $\sim 26$ weeks GA and then decrease (Jammes and Gilles, 1983), which supports the pattern seen in this analysis. Also by 20 weeks GA, the basic organization of supragranular and infragranular layers is present in the cortical plate, and further proliferation of cortical neural precursor cells is limited to the subventricular zone of the germinal matrix [ for review, see Rakic (1988) and Bystron et al. (2008)]. The diminishing proliferation in the germinal matrix during our period of study may explain the slower growth.

\section{Future directions}

Motion correction and 3D reconstruction of in utero multislice 2D T2w MRI provide a geometrically consistent $3 \mathrm{D}$ volumetric image of the entire brain, and enable quantitative normal brain growth studies that complement postmortem histological studies of the human fetus. Although our analysis is now based on a relatively small number of fetuses, compared to postnatal imaging studies, we have established the applicability and strength of our method for early fetal imaging studies. By using our TBM approach, definitive patterns of spatial variations in brain growth and maturation rates may be obtained as more normal fetal images become available. Due to the ethical considerations of repeated in utero fetal MRI and the necessary short intervals between imaging sessions, we were not able to conduct longitu- dinal analysis to look at individual growth patterns and reduce intersubject variability. However, the morphological changes over this age range far outweigh intersubject differences in the fetal brain before the emergence of tertiary sulci. These normative profiles of change in volume derived from cross-sectional data may quantify the normal variation and distinguish abnormal brain development from random intersubject variations.

Though in the present study we solely used the volume information from the deformation tensor analysis, additional parameters can represent other aspects of the growth patterns. For example, studying directional, volume-preserving changes in tissue growth (Lepore et al., 2008) may complement the findings presented in this study.

Anatomical interpretations of the growth rate maps derived from the nonlinear registration are limited to the available tissue boundaries - in this study, CP, NCP, and VENT. Further refinements of these maps will be possible with the development of registration methods capable of dealing with transient tissue classes, such as the germinal matrix and subplate, and making use of additional tissue contrasts such as DTI (Studholme, 2008).

\section{Conclusion}

This was the first application of TBM to in utero structural MRI of normally developing fetal brains and appends to the age range of the growing body of work characterizing growth patterns in children, term neonates, and prematurely born infants (Hüppi et al., 1998; Giedd et al., 1999; Sowell et al., 2003; Aljabar et al., 2008; Dubois et al., 2008; Knickmeyer et al., 2008; Hua et al., 2009). By this method, we have identified where tissue volume and shape changes are deviating from the global trajectory between 20 and 28 weeks GA. We have detected the emergence of sulci and gyri by differentiating between the changes in the cortical plate and the underlying cerebral mantle. Extension of this type of analysis to later fetal ages or postnatally may characterize further cortical folding and myelination that is associated with anatomical changes in T1w, T2w, and diffusion-weighted MRI.

\section{References}

Aljabar P, Bhatia KK, Murgasova M, Hajnal JV, Boardman JP, Srinivasan L, Rutherford MA, Dyet LE, Edwards AD, Rueckert D (2008) Assessment of brain growth in early childhood using deformation-based morphometry. Neuroimage 39:348-358.

Bendersky M, Musolino PL, Rugilo C, Schuster G, Sica REP (2006) Normal anatomy of the developing fetal brain. Ex vivo anatomical-magnetic resonance imaging correlation. J Neurol Sci 250:20-26.

Boardman JP, Counsell SJ, Rueckert D, Kapellou O, Bhatia KK, Aljabar P, Hajnal J, Allsop JM, Rutherford MA, Edwards AD (2006) Abnormal deep grey matter development following preterm birth detected using deformation-based morphometry. Neuroimage 32:70-78.

Bystron I, Blakemore C, Rakic P (2008) Development of the human cerebral cortex: Boulder committee revisited. Nat Rev Neurosci 9:110-122.

Cardenas VA, Studholme C, Gazdzinski S, Durazzo TC, Meyerhoff DJ (2007) Deformation-based morphometry of brain changes in alcohol dependence and abstinence. Neuroimage 34:879-887.

Christensen G, Rabbitt R, Miller M (1996) Deformable templates using large-deformation kinematics. IEEE Trans Image Process 5:1435-1447.

Chung MK, Worsley KJ, Paus T, Cherif C, Collins DL, Giedd JN, Rapoport JL, Evans AC (2001) A unified statistical approach to deformation-based morphometry. Neuroimage 14:595-606.

Davatzikos C, Vaillant M, Resnick SM, Prince JL, Letovsky S, Bryan RN (1996) A computerized approach for morphological analysis of the corpus callosum. J Comput Assist Tomogr 20:88-97.

Diaz AL, Gleeson JG (2009) The molecular and genetic mechanisms of neocortex development. Clin Perinatol 36:503-512.

Dooling EC, Chi JG, Gilles FH (1983) Telencephalic development: changing gyral patterns. In: The developing human brain: growth and epidemio- 
logic neuropathology (Gilles F, Leviton A, Dooling E, eds), pp 94-104. Boston, MA: John Wright-PSC.

Dubois J, Benders M, Cachia A, Lazeyras F, Ha-Vinh Leuchter R, Sizonenko SV, Borradori-Tolsa C, Mangin JF, Hüppi PS (2008) Mapping the early cortical folding process in the preterm newborn brain. Cereb Cortex 18:1444-1454.

Garel C, Chantrel E, Elmaleh M, Brisse H, Sebag G (2003) Fetal MRI: normal gestational landmarks for cerebral biometry, gyration and myelination. Childs Nerv Syst 19:422-425.

Giedd JN, Blumenthal J, Jeffries NO, Castellanos FX, Liu H, Zijdenbos A, Paus T, Evans AC, Rapoport JL (1999) Brain development during childhood and adolescence: a longitudinal MRI study. Nat Neurosci 2:861-863.

Glenn OA, Barkovich J (2006) Magnetic resonance imaging of the fetal brain and spine: an increasingly important tool in prenatal diagnosis, Part 2. AJNR Am J Neuroradiol 27:1807-1814.

Grossman R, Hoffman C, Mardor Y, Biegon A (2006) Quantitative MRI measurements of human fetal brain development in utero. Neuroimage 33:463-470.

Guibaud L, Selleret L, Larroche JC, Buenerd A, Alias F, Gaucherand P, Des Portes V, Pracros JP (2008) Abnormal Sylvian fissure on prenatal cerebral imaging: significance and correlation with neuropathological and postnatal data. Ultrasound Obstet Gynecol 32:50-60.

Habas PA, Kim K, Corbett-Detig JM, Rousseau F, Glenn OA, Barkovich AJ, Studholme C (2010a) A spatiotemporal atlas of MR intensity, tissue probability and shape of the fetal brain with application to segmentation. Neuroimage 53:460-470.

Habas PA, Kim K, Rousseau F, Glenn OA, Barkovich AJ, Studholme C (2010b) Atlas-based segmentation of developing tissues in the human brain with quantitative validation in young fetuses. Hum Brain Mapp 31:1348-1358.

Hilgetag CC, Barbas H (2006) Role of mechanical factors in the morphology of the primate cerebral cortex. PLoS Comput Biol 2:e22.

Hu HH, Guo WY, Chen HY, Wang PS, Hung CI, Hsieh JC, Wu YT (2009) Morphological regionalization using fetal magnetic resonance images of normal developing brains. Eur J Neurosci 29:1560-1567.

Hua X, Leow AD, Levitt JG, Caplan R, Thompson PM, Toga AW (2009) Detecting brain growth patterns in normal children using tensor-based morphometry. Hum Brain Mapp 30:209-219.

Hüppi PS, Warfield S, Kikinis R, Barnes PD, Zientara GP, Jolesz FA, Tsuji MK, Volpe JJ (1998) Quantitative magnetic resonance imaging of brain development in premature and mature newborns. Ann Neurol 43:224-235.

Jammes JL, Gilles FH (1983) Telencephalic development: matrix volume and isocortex and allocortex surface areas. In: The developing human brain: growth and epidemiologic neuropathology (Gilles F, Leviton A, Dooling E, eds), pp 87-93. Boston, MA: John Wright-PSC.

Jiang S, Xue H, Glover A, Rutherford M, Rueckert D, Hajnal JV (2007) MRI of moving subjects using multislice snapshot images with volume reconstruction (SVR): application to fetal, neonatal, and adult brain studies. IEEE Trans Med Imaging 26:967-980.

Kazan-Tannus JF, Dialani V, Kataoka ML, Chiang G, Feldman HA, Brown JS, Levine D (2007) MR volumetry of brain and CSF in fetuses referred for ventriculomegaly. AJR Am J Roentgenol 189:145-151.

Kim K, Habas PA, Rousseau F, Glenn OA, Barkovich AJ, Studholme C (2010) Intersection based motion correction of multislice MRI for 3-D in utero fetal brain image formation. IEEE Trans Med Imaging 29:146-158.

Kinoshita Y, Okudera T, Tsuru E, Yokota A (2001) Volumetric analysis of the germinal matrix and lateral ventricles performed using $\mathrm{mr}$ images of postmortem fetuses. AJNR Am J Neuroradiol 22:382-388.

Knickmeyer RC, Gouttard S, Kang C, Evans D, Wilber K, Smith JK, Hamer RM, Lin W, Gerig G, Gilmore JH (2008) A structural MRI study of human brain development from birth to 2 years. J Neurosci 28: 12176-12182.

Kochunov P, Castro C, Davis D, Dudley D, Brewer J, Zhang Y, Kroenke CD, Purdy D, Fox PT, Simerly C, Schatten G (2010) Mapping primary gyrogenesis during fetal development in primate brains: high-resolution in utero structural MRI of fetal brain development in pregnant baboons. Front Neurosci 4:20.

Kostović I, Judas M (2010) The development of the subplate and thalamocortical connections in the human foetal brain. Acta Paediatr 99:1119-1127.

Kostovic I, Rakic P (1990) Developmental history of the transient subplate zone in the visual and somatosensory cortex of the macaque monkey and human brain. J Comp Neurol 297:441-470.
LeMay M (1976) Morphological cerebral asymmetries of modern man, fossil man, and nonhuman primate. Ann N Y Acad Sci 280:349-366.

Lepore N, Brun C, Chou YY, Chiang MC, Dutton RA, Hayashi KM, Luders E, Lopez OL, Aizenstein HJ, Toga AW, Becker JT, Thompson PM (2008) Generalized tensor-based morphometry of HIV/AIDS using multivariate statistics on deformation tensors. IEEE Trans Med Imaging 27:129-141.

Lopes A, Brodlie K (2003) Improving the robustness and accuracy of the marching cubes algorithm for isosurfacing. IEEE Trans Vis Comput Graph 9:16-29.

Modersitzki J (2004) Numerical methods for image registration. Oxford: Oxford UP.

Nichols TE, Holmes AP (2002) Nonparametric permutation tests for functional neuroimaging: a primer with examples. Hum Brain Mapp 15:1-25.

Pieperhoff P, Hömke L, Schneider F, Habel U, Shah NJ, Zilles K, Amunts K (2008) Deformation field morphometry reveals age-related structural differences between the brains of adults up to 51 years. J Neurosci 28:828-842.

Quarello E, Stirnemann J, Ville Y, Guibaud L (2008) Assessment of fetal Sylvian fissure operculization between 22 and 32 weeks: a subjective approach. Ultrasound Obstet Gynecol 32:44-49.

Rakic P (1988) Specification of cerebral cortical areas. Science 241:170-176.

Rousseau F, Glenn OA, Iordanova B, Rodriguez-Carranza C, Vigneron DB, Barkovich JA, Studholme C (2006) Registration-based approach for reconstruction of high-resolution in utero fetal MR brain images. Acad Radiol 13:1072-1081.

Smart IH, McSherry GM (1986a) Gyrus formation in the cerebral cortex in the ferret. I. Description of the external changes. J Anat 146:141-152.

Smart IH, McSherry GM (1986b) Gyrus formation in the cerebral cortex of the ferret. II. Description of the internal histological changes. J Anat 147:27-43.

Sowell ER, Peterson BS, Thompson PM, Welcome SE, Henkenius AL, Toga AW (2003) Mapping cortical change across the human life span. Nat Neurosci 6:309-315.

Studholme C (2008) Dense feature deformation morphometry: incorporating DTI data into conventional MRI morphometry. Med Image Anal 12:742-751.

Studholme C, Cardenas V (2004) A template free approach to volumetric spatial normalization of brain anatomy. Pattern Recognit Lett 25:1191-1202.

Studholme C, Hill DLG, Hawkes DJ (1999) An overlap invariant entropy measure of 3D medical image alignment. Pattern Recognit 32:71-86.

Studholme C, Cardenas V, Schuff N, Rosen H, Miller B, Weiner M (2001) Detecting spatially consistent structural differences in Alzheimer's and fronto temporal dementia using deformation morphometry. In: Medical image computing and computer-assisted intervention-MICCAI 2001 (Niessen WJ, Viergever MA, eds), pp 41-48. New York: Springer.

Studholme C, Cardenas V, Blumenfeld R, Schuff N, Rosen HJ, Miller B, Weiner M (2004) Deformation tensor morphometry of semantic dementia with quantitative validation. Neuroimage 21:1387-1398.

Thirion JP (1998) Image matching as a diffusion process: an analogy with Maxwell's demons. Med Image Anal 2:243-260.

Thompson PM, Giedd JN, Woods RP, MacDonald D, Evans AC, Toga AW (2000) Growth patterns in the developing brain detected by using continuum mechanical tensor maps. Nature 404:190-193.

Thompson PM, Mega MS, Woods RP, Zoumalan CI, Lindshield CJ, Blanton RE, Moussai J, Holmes CJ, Cummings JL, Toga AW (2001) Cortical change in Alzheimer's disease detected with a disease-specific populationbased brain atlas. Cereb Cortex 11:1-16.

Toga AW, Thompson PM (2003) Mapping brain asymmetry. Nat Rev Neurosci 4:37-48.

Toi A, Lister WS, Fong KW (2004) How early are fetal cerebral sulci visible at prenatal ultrasound and what is the normal pattern of early fetal sulcal development? Ultrasound Obstet Gynecol 24:706-715.

Tzarouchi LC, Astrakas LG, Xydis V, Zikou A, Kosta P, Drougia A, Andronikou S, Argyropoulou MI (2009) Age-related grey matter changes in preterm infants: an MRI study. Neuroimage 47:1148-1153.

Viola P, Wells WM (1997) Alignment by maximization of mutual information. Int J Comput Vis 24:137-154

Widjaja E, Geibprasert S, Mahmoodabadi SZ, Blaser S, Brown NE, Shannon P (2010) Alteration of human fetal subplate layer and intermediate zone during normal development on $\mathrm{mr}$ and diffusion tensor imaging. AJNR Am J Neuroradiol 31:1091-1099. 